\title{
O Uso do Gráfico de Resíduos no Modelamento
}

\section{de Dados Químicos}

Aline Renée Coscione

Audrey de Arruda Falcão

João Carlos de Andrade *

dandrade@iqm.unicamp.br

Universidade Estadual de Campinas, Instituto de Química

\section{Informações do Artigo}

Histórico do Artigo

Criado em Abril de 2006

Palavras-Chaves

Superfície de respostas

Análise de variância

ANOVA

Otimização multivariada

Gráfico de resíduos

Planejamento experimental modelamento
Resumo

Nesse trabalho empregou-se o método da análise de superfície de reposta para otimizar a resposta catalítica do $\mathrm{Mo}(\mathrm{VI})$ na reação de oxidação de íons iodeto pela água oxigenada, obtidas pelo método do tempo fixo. A absorbância da reação não catalítica e da ação catalítica do $\mathrm{Mo}(\mathrm{VI})$, o delta, foram modeladas. Isso foi feito de forma detalhada para ilustrar a importância da avaliação cuidadosa dos resultados, o que nem sempre é destacado devido ao rigor matemático necessário e à dificuldade dos usuários das técnicas multivariadas em lidar com essa linguagem.

O exemplo apresentado neste trabalho mostra que apenas a análise de variância (ANOVA) não é um critério suficiente para a seleção de um modelo, que pode descrever um determinado conjunto de dados, sendo imprescindível que a análise do gráfico dos resíduos também seja feita, mantendo-se uma visão crítica dos resultados obtidos.

Os vinte e quatro ensaios do planejamento fatorial inicialmente empregados no modelamento (doze na ausência e doze na presença de molibdênio), e posteriormente estendidos a um planejamento estrela (oito ensaios), podem ser realizados numa aula experimental de 4 a 6 horas, permitindo sua inclusão num curso experimental para a graduação, introduzindo o uso de métodos multivariados de otimização.

O emprego de um procedimento de extração do iodo gerado in situ permite ainda "congelar" a reação catalítica num tempo de reação pré-determinado, permitindo a realização de um número elevado de experimentos de maneira eficiente através do processo em batelada, tornando possível a obtenção dos dados necessários à construção dos modelos matemáticos.

\section{Objetivo}

Ilustrar a importância da análise criteriosa do gráfico de resíduos do modelamento versus as respostas dos ensaios realizados, usando planejamentos experimentais previamente estabelecidos, na seleção de modelos que descrevam adequadamente as respostas experimentais. 


\section{Introdução}

Técnicas de otimização de reações químicas são de grande valor em Química Analítica, pois podem indicar condições operacionais que favorecem a determinação de compostos ou elementos de interesse numa amostra. Isso pode ser alcançado por meio do aumento da sensibilidade do método empregado, o que muitas vezes é evidenciado por um aumento da propriedade que está sendo medida (por exemplo, absorbância ou corrente), ou pelo aumento de seletividade, selecionando-se condições em que a resposta devido a espécies interferentes seja minimizada.

O método de otimização mais empregado, intuitivo para a maioria das pessoas, é o método no qual variase um fator de cada vez, fixando-se os demais (método univariado). Porém este método apresenta uma série de desvantagens e vem sendo gradativamente substituído, por métodos multivariados, nos quais diversos fatores são variados simultaneamente [1]. Isso não ocorre apenas por que, em geral, um número menor de experimentos é necessário para encontrar as condições de operacionais mais favoráveis, mas principalmente por que se ocorrerem interações entre os fatores experimentais e esses efeitos forem significativos quando comparadas com o erro experimental, isso será considerado na definição da região de ótimo. Outra vantagem, é a possibilidade de construir modelos matemáticos que descrevem o comportamento dos fatores experimentais a partir do planejamento experimental utilizado. Isso torna possível realizar previsões sobre os valores da resposta modelada dentro da região em que os fatores foram variados, em oposição à resposta pontual obtida no método univariado.

Além disso, em alguns casos, o emprego de uma abordagem estatística aliada à interpretação química passa a ser essencial para descrever e compreender sistemas químicos aparentemente simples. Um exemplo claro é o modelamento da resposta espectrofotométrica das reações de complexação de tiocianato com $\mathrm{Co}(\mathrm{II})$ ou $\mathrm{Fe}(\mathrm{III})$, quando o meio reacional é constituído por um sistema ternário homogêneo de solventes [2]. Em situações como esta o uso de métodos multivariados tornam-se particularmente importantes em condições industriais e de análise de rotina, pois implicam em tempo e custos, fazendo com que seu uso seja preferido nestas aplicações.

Porém, embora os métodos multivariados de otimização estejam se tornando mais populares, a obtenção de modelos satisfatórios e bem ajustados requer não apenas a realização de planejamentos experimentais sob o olhar cuidadoso do químico, mas também a capacidade de analisar criticamente os resultados obtidos nas diversas etapas de cálculos envolvidas. Isso é decisivo para a adequada seleção de modelos e avaliação dos erros calculados.

A metodologia de superfícies de resposta envolve o uso de planejamentos fatoriais que podem ser repetidos várias vezes, mapeando a superfície de respostas obtidas na direção da região ponto de ótimo desejado [1-4]. Outro detalhe importante é o uso das variáveis em sua forma escalonada, de forma que suas grandezas não interfiram no desenvolvimento do processo de otimização.

A oxidação de íons iodeto por peróxido de hidrogênio em meio ácido tem sido aplicada na determinação de molibdênio em diversas matrizes, empregando tanto o processo em batelada [5,6] quanto sistemas em fluxo $[7,8]$. Esses métodos empregam a técnica do tempo fixo, na qual registra-se a absorbância do produto da reação, na região da luz visível, num tempo pré-determinado após o início da reação. As leituras de absorbância podem ser feitas no comprimento de onda de absorção do iodo gerado, ou pode-se adicionar uma solução de amido ao meio, que complexa o iodo, produzindo um complexo azulado que aumenta a sensibilidade das determinações. Essa alternativa é usualmente empregada nos processos em batelada.

No entanto, como a reação de oxidação de íons iodeto pela água oxigenada ocorre naturalmente, é importante lembrar que a absorbância registrada nos experimentos na presença do catalisador corresponde à soma da reação não catalítica e da catalítica já que a primeira não pode ser impedida. Como o que se deseja otimizar é o método cinético de determinação de íons $\mathrm{Mo}(\mathrm{VI})$, ou seja é a reação promovida pelo catalisador, torna-se necessário definir uma reposta analítica de interesse, aqui denominada delta $(\Delta \mathrm{A})$. O delta correspondente à diferença das absorbâncias entre a reação não catalítica (de ocorrência natural) e da reação na presença do catalisador (não catalítica mais catalítica).

Neste experimento, além do delta modelou-se também a reação não catalítica para tentar definir a condição de ótimo de determinação do $\mathrm{Mo}(\mathrm{VI})$, numa situação em que a absorbância na reação não catalítica seja mínima. Isso é importante pois num método analítico empregado para a quantificação de uma espécie de interesse deseja-se que as condições operacionais empregadas sejam as que resultam na maior sensibilidade possível. Neste caso, isso implica na maior diferença entre o sinal analítico (nesse caso a absorbância) entre a reação que ocorre naturalmente (reação não catalítica) e a reação obtida na presença do catalisador. 


\section{Parte Experimental}

\section{Reagentes e Materiais}

Os materiais e reagentes necessários para esse experimento são:

- Solução padrão de molibdênio

- Peróxido de hidrogênio

- Iodeto de potássio

- Ácido sulfúrico concentrado

- Água deionizada

- Espectrofotômetro

- Balança analítica

- Micropipetas

- Pipetas volumétricas

- Buretas

- Balões volumétricos

- Béqueres

- Funis de separação de $60,0 \mathrm{~mL}$

- Espátulas

\section{Preparação das soluções}

Todas as soluções foram preparadas com água deionizada e estocadas em frascos de polietileno limpos. As transferências de volume foram feitas utilizandose micropipetas, pipetas volumétricas ou buretas, dependendo do volume utilizado. As reações e medidas espectrofotométricas foram realizadas mantendo-se uma temperatura ambiente de $23 \pm 20 \mathrm{C}$.

Preparou-se uma solução 1000,0 mg L $\mathrm{L}^{-1}$ de molibdênio através da diluição do conteúdo de uma ampola de padrão Merck - Titrisol, para um litro, com água deionizada. A partir dessa solução estoque, preparou-se uma solução de trabalho de $1,0 \mathrm{mg} \mathrm{L}^{-1}$ de molibdênio, a partir da qual foram tomadas alíquotas apropriadas para a realização dos experimentos.

Uma solução 5,0 × $10^{-2} \mathrm{~mol} \mathrm{~L}^{-1}$ de peróxido de hidrogênio foi preparada diariamente pela diluição da solução comercial concentrada $(30 \% \mathrm{~m} / \mathrm{m})$, a qual foi padronizada por titulação [9]. A solução $5,0 \times 10^{-3} \mathrm{~mol} \mathrm{~L}^{-1}$ de iodeto de potássio foi preparada pela dissolução da massa apropriada do sal em água. Um volume suficientemente grande dessa solução foi preparado para ser utilizado em todos os experimentos, tornando desnecessária sua padronização. Uma solução 1,0 $\mathrm{mol} \mathrm{L}^{-1}$ de ácido sulfúrico foi preparada por diluição do ácido concentrado.
Realização das medidas

Os íons triiodeto, posteriormente extraídos com clorofórmio, foram gerados pela mistura dos reagentes em funis de separação de $60,0 \mathrm{~mL}$, previamente limpos e secos, com torneiras de Teflon. O processo é realizado em batelada e as medidas de absorbância são realizadas num tempo fixo, correspondente a um tempo de reação de 20 minutos. Para permitir o modelamento de $\Delta \mathrm{A}$, para cada composição de reagentes, como aquela mostradas na Tabela 1, realizaram-se 2 experimentos: o primeiro com a adição de uma concentração fixa de 40,0 $\mu \mathrm{g} \mathrm{L}^{-1} \mathrm{de} \mathrm{Mo}(\mathrm{VI})$, correspondente a 2,0 mL da solução de trabalho $1,0 \mathrm{mg}$ $\mathrm{L}^{-1}$, e o segundo com a adição de uma alíquota de água deionizada para substituir o molibdênio, correspondentes às reações catalíticas e não catalíticas, respectivamente.

As concentrações e os volumes dos reagentes adicionados foram calculados para atingir um volume final de 50,0 mL, completado pela adição de água deionizada quando necessário. Como a função principal da água é a padronização dos volumes, essa pode ser adicionada quando for mais conveniente, assim a ordem de adição dos reagentes foi sempre mantida a seguinte: água (necessária para atingir 50,0 mL), ácido sulfúrico, peróxido de hidrogênio, molibdênio (se a reação for catalítica) e finalmente o iodeto de potássio. Depois da adição da solução do iodeto de potássio a solução foi homogeneizada e o cronômetro acionado. Após decorridos os 20 minutos, adicionou-se $5,0 \mathrm{~mL}$ de clorofórmio e os funis foram agitados vigorosamente durante 1 minuto. Então a fração orgânica foi transferida para cubetas de quartzo e a absorbância do iodo contido nessa fase foi lida em 510 nm.

\section{Agradecimentos}

O planejamento experimental empregado na primeira etapa da otimização consistiu num planejamento fatorial $2^{3}$ com ponto central. As variáveis estudadas foram a concentração de iodeto de potássio, água oxigenada e ácido sulfúrico, correspondentes às variáveis escalonadas $\mathrm{x}_{1}, \mathrm{x}_{2}$ e $\mathrm{x}_{3}$, respectivamente. Foram realizadas 3 replicatas do ponto central, num total de 11 experimentos. Os níveis mínimo e máximo de concentração selecionados são os empregados em estudos anteriores (dados não publicados) e correspondem a concentrações entre 1 e $3 \mathrm{mmol} \mathrm{L}^{-1}$ de $\mathrm{KI}\left(\mathrm{C}_{\mathrm{KI}}\right) ; 0,5$ e $1 \mathrm{mmol} \mathrm{L}^{-1}$ de $\mathrm{H}_{2} \mathrm{O}_{2}\left(\mathrm{C}_{\mathrm{H} 2 \mathrm{O} 2}\right)$ e 0,02 e $0,06 \mathrm{~mol} \mathrm{~L}^{-1}$ de $\mathrm{H}_{2} \mathrm{SO}_{4}\left(\mathrm{C}_{\mathrm{H} 2 \mathrm{SO}}\right)$. No entanto, como será discutido a seguir, esse planejamento foi transformado posteriormente num planejamento estrela, através da 
Tabela 1. Planejamento estrela utilizado da coleta de dados para o modelamento*.

\begin{tabular}{|c|c|c|c|c|c|c|c|c|c|}
\hline \multirow[b]{3}{*}{ Ensaio } & \multicolumn{3}{|l|}{ Variáveis } & & & & & & \\
\hline & \multirow{2}{*}{$\frac{\mathrm{C}_{\mathrm{KI}}}{\mathrm{mmol} / \mathrm{L}}$} & \multirow{2}{*}{$\frac{\mathrm{C}_{\mathrm{H} 2 \mathrm{O} 2}}{\mathrm{mmol} / \mathrm{L}}$} & \multirow{2}{*}{$\begin{array}{l}\mathrm{C}_{\mathrm{H} 2 \mathrm{SO} 4} \\
\mathrm{mmol} / \mathrm{L}\end{array}$} & \multicolumn{3}{|c|}{$\begin{array}{l}\text { Concentrações } \\
\text { escalonadas }^{* *}\end{array}$} & \multicolumn{3}{|c|}{ Absorbância (Abs) } \\
\hline & & & & $\mathrm{x}_{1}$ & $\mathbf{x}_{2}$ & $\mathbf{x}_{3}$ & $\begin{array}{l}\text { Não } \\
\text { catalítica }\end{array}$ & Catalítica & Delta \\
\hline 1 & 1,0 & 0,5 & 0,02 & -1 & -1 & -1 & 0,08 & 0,47 & 0,39 \\
\hline 2 & 3,0 & 0,5 & 0,02 & +1 & -1 & -1 & 0,26 & 1,24 & 0,99 \\
\hline 3 & 1,0 & 1,0 & 0,02 & -1 & +1 & -1 & 0,20 & 0,68 & 0,48 \\
\hline 4 & 3,0 & 1,0 & 0,02 & +1 & +1 & -1 & 0,40 & 1,64 & 1,24 \\
\hline 5 & 1,0 & 0,5 & 0,06 & -1 & -1 & +1 & 0,12 & 0,59 & 0,47 \\
\hline 6 & 3,0 & 0,5 & 0,06 & +1 & -1 & +1 & 0,30 & 1,28 & 0,98 \\
\hline 7 & 1,0 & 1,0 & 0,06 & -1 & +1 & +1 & 0,29 & 0,75 & 0,47 \\
\hline 8 & 3,0 & 1,0 & 0,06 & +1 & +1 & +1 & 0,53 & 1,84 & 1,31 \\
\hline 9 & 2,0 & 0,75 & 0,04 & 0 & 0 & 0 & 0,25 & 1,14 & 0,89 \\
\hline 10 & 2,0 & 0,75 & 0,04 & 0 & 0 & 0 & 0,34 & 1,16 & 0,82 \\
\hline 11 & 2,0 & 0,75 & 0,04 & 0 & 0 & 0 & 0,33 & 1,18 & 0,85 \\
\hline 12 & 2,0 & 0,75 & 0,04 & 0 & 0 & 0 & 0,25 & 1,20 & 0,94 \\
\hline 13 & 0,6 & 0,75 & 0,04 & $-1,41$ & 0 & 0 & 0,12 & 0,41 & 0,29 \\
\hline 14 & 3,4 & 0,75 & 0,04 & 1,41 & 0 & 0 & 0,46 & 1,70 & 1,24 \\
\hline 15 & 2,0 & 0,4 & 0,04 & 0 & $-1,41$ & 0 & 0,18 & 0,81 & 0,63 \\
\hline 16 & 2,0 & 1,1 & 0,04 & 0 & 1,41 & 0 & 0,47 & 1,29 & 0,82 \\
\hline 17 & 2,0 & 0,75 & 0,012 & 0 & 0 & $-1,41$ & 0,22 & 1,06 & 0,84 \\
\hline 18 & 2,0 & 0,75 & 0,068 & 0 & 0 & 1,41 & 0,40 & 1,29 & 0,89 \\
\hline
\end{tabular}

* Para permitir o modelamento do $\Delta \mathrm{A}$, para cada composição de reagentes, realizaram-se 2 experimentos: o primeiro com a adição de $2,0 \mathrm{~mL}$ de uma solução $1 \mathrm{mg} \mathrm{L}^{-1}$, para manter uma concentração fixa de $40 \mu \mathrm{g} \mathrm{L}{ }^{-1}$ de $\mathrm{Mo}(\mathrm{VI})$ e o segundo com a adição de uma alíquota de água deionizada para substitutir o molibdênio, correspondentes às reações catalíticas e não catalíticas. $\mathrm{O} \Delta \mathrm{A}$ é obtido pela diferença de absorbância entre esses dois ensaios.

** Os escalonamentos estão descritos nas equações 1-3, p.6.

adição de uma replicata no ponto central e dos níveis 1,41 e $-1,41$, correspondentes aos seis últimos experimentos mostrados na Tabela 1.

Assim, o escalonamento das variáveis estudadas é obtido usando as equações de 1 a 3 :

$$
\begin{aligned}
& \mathrm{x}_{1}=\left(\mathrm{C}_{\mathrm{KI}}-2\right) / 1 \\
& \mathrm{x}_{2}=\left(\mathrm{C}_{\mathrm{H} 2 \mathrm{O} 2}-0,75\right) / 0,25 \\
& \mathrm{x}_{3}=\left(\mathrm{C}_{\mathrm{H} 2 \mathrm{SO} 4}-0,04\right) / 0,02
\end{aligned}
$$

equação 1

equação 2

equação 3

\section{O modelamento}

Nossa otimização começa pelo modelamento da reação não catalítica, empregando os valores de absorbância listados na Tabela 1. Os valores de absorbância obtidos e a composição dos ensaios, empregando as variáveis escalonadas, devem ser agrupados em matrizes para a realização dos cálculos, que é também o formato utilizado através do programa freeware MODREG disponibilizado no site Chemkeys. As instruções para utilização do programa também estão disponíveis no endereço acima.

Mesmo sem a utilização do programa sugerido, a ordem de entrada dos dados deve obedecer rigorosamente àquela mostrada na Tabela 1, também conhecida como ordem padrão do planejamento, para permitir a realização das operações com matrizes envolvidas na resolução de mínimos quadrados parciais. A saída do programa inclui, entre outras coisas, a análise de variância (ANOVA) para o ajuste do modelo selecionado aos dados e, como em qualquer modelamento, é necessário verificar primeiramente se a regressão é significativa e se existe evidência de falta de ajuste [3].

Os cálculos necessários para a construção e a avaliação dos modelos foram feitos usando a resolução de mínimos 
quadrados parciais. A ANOVA para o modelo linear da reação não catalítica é apresentada na Tabela 2.

Tabela 2. Análise da variância para o ajuste do modelo linear aos dados de absorbância para a reação não catalítica, a partir dos ensaios 1 a 11 da Tabela 1.

\begin{tabular}{|l|l|l|l|l|}
\hline $\begin{array}{l}\text { Fonte de } \\
\text { Variação }\end{array}$ & $\begin{array}{l}\text { Soma } \\
\text { Quadrática }\end{array}$ & $\begin{array}{l}\text { Graus de } \\
\text { Liberdade }\end{array}$ & $\begin{array}{l}\text { Média } \\
\text { Quadrática }\end{array}$ & Teste F \\
\hline Regressão & 0,1456 & 3 & 0,0485 & \\
\hline Resíduos & 0,0108 & 8 & 0,0014 & 35,85 \\
\hline $\begin{array}{l}\text { Façta de } \\
\text { ajuste }\end{array}$ & 0,0045 & 5 & 0,0009 & \\
\hline Erro puro & 0,0064 & 3 & 0,0021 & 0,42 \\
\hline Total & 0,1564 & 11 & & \\
\hline \% da variância $=93,07$ & \multicolumn{3}{|l}{} \\
\hline
\end{tabular}

$\%$ máxima de variância explicada $=95,93$

A partir desses resultados verifica-se que a regressão é significativa na descrição dos resultados experimentais, e que o modelo linear aparentemente é adequado, pois não há evidência de falta de ajuste, pois para um nível de confiança de $95 \%$, os valores tabelados do teste F para (3, 8) e $(5,3)$ são, respectivamente, de 4,07 e 9,01.

Porém, o exame do gráfico de resíduos do modelo, mostrado na Figura 1, não apresenta-se com uma distribuição aleatória, como seria esperado para um modelo bem ajustado. Ao contrário, pode-se observar a existência de uma tendência nos resíduos. Isso levanta dúvidas sobre a adequação do modelo testado, pois pode indicar a presença de um efeito das variáveis sobre a resposta experimental que não está sendo adequadamente modelado. Neste caso, deve-se verificar o ajuste de um modelo mais complexo, no caso, o modelo quadrático. Para isso são necessários mais experimentos, já que um modelo quadrático para três variáveis pode ter até 9 termos significativos $[1,3]$.

A maneira mais simples para obter o número de experimentos necessários é transformar o planejamento fatorial empregado num planejamento estrela. Adicionouse então uma replicata do ponto central (ensaio 12) e os experimentos de 13 a 16, que correspondem ao planejamento estrela. Uma explicação mais detalhada sobre este tipo de planejamento pode ser encontrada na literatura $[3,4]$.

A ANOVA para a avaliação do ajuste do modelo quadrático para a reação não catalítica para todos os ensaios listados da Tabela 1 é mostrada na Tabela 3. Mais uma vez, para um nível de confiança de $95 \%$, verifica-se que a regressão é significativa na descrição dos resultados, e que o modelo não apresenta falta de ajuste.

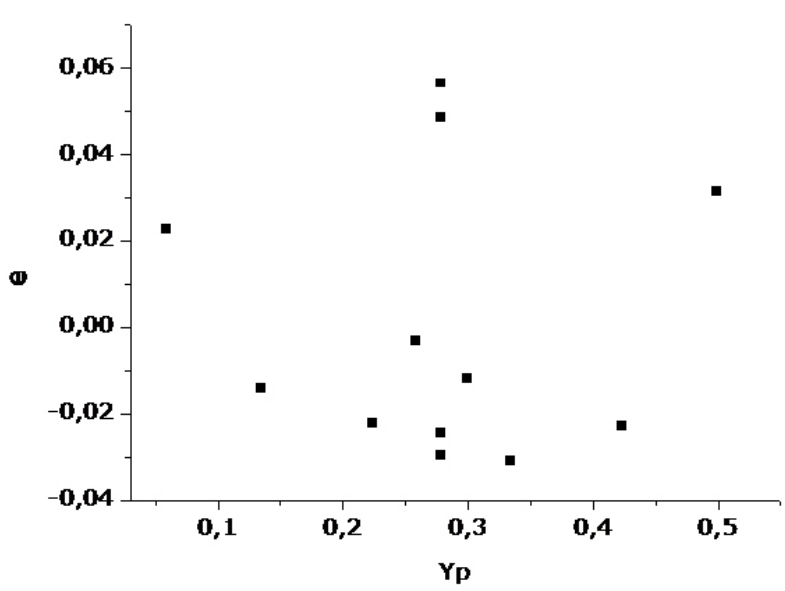

Figura 1. Gráfico dos resíduos (e) contra as respostas previstas (Yp) para a reação não catalítica-Modelo linear.

No entanto, é necessário verificar quais efeitos são de fato significativos, ou seja, aumentam a produção de iodo da reação e consequentemente aumentam a absorbância medida, distinguindo-se da variação aleatória existente em qualquer experimento. Para que isso seja possível é necessário admitir uma série de hipóteses estatísticas, que podem ser aceitas em problemas quando os experimentos são realizados de forma aleatória e de forma cuidadosa [3]. Nessas condições, os valores que permitem a distinção entre efeito e ruído serão uma função do planejamento experimental empregado (refletido no erro padrão dos coeficientes), da variação entre as replicatas realizadas e do nível de confiança estatística empregado, e a matriz de covariância dada por $\mathbf{V}(\mathbf{b})=\left(\mathbf{X}^{\mathbf{t}} \mathbf{X}\right)^{-1} \mathbf{s}^{2}$ pode ser empregada nos cálculos, sendo $\mathrm{X}$ a matriz de planejamento (colunas das variáveis codificadas da Tabela 1) e $\mathrm{s}^{2}$ a estimativa da variância populacional.

Além disso, como não há evidência de falta de ajuste, a Média Quadrática dos resíduos para ajuste do modelo quadrático, mostrada na ANOVA da Tabela 3, que corresponde à variância residual em torno da regressão pode ser empregada como uma estimativa de $\mathrm{s}^{2}$. Pode-se calcular então o erro padrão dos coeficientes do modelo, multiplicando-se os valores da diagonal principal da matriz de covariância pela referida média quadrática e obtendo-se sua raiz quadrada [3].

A equação 4, apenas com os efeitos significativos, representa o modelo resultante. Os valores entre parênteses indicam o erro padrão dos coeficientes.

$$
\begin{gathered}
A=0,30( \pm 0,02)+0,11 x_{1}( \pm 0,01)+0,09 x_{2}( \pm 0,01)+ \\
+0,05 x_{3}( \pm 0,01) \quad \text { equação } 4
\end{gathered}
$$


Tabela 3. Análise de variância para o ajuste do modelo quadrático para a reação não catalítica aos dados do planejamento estrela.

\begin{tabular}{|l|l|l|l|l|}
\hline $\begin{array}{l}\text { Fonte de } \\
\text { Variação }\end{array}$ & $\begin{array}{l}\text { Soma } \\
\text { Quadrática }\end{array}$ & $\begin{array}{l}\text { Graus de } \\
\text { Liberdade }\end{array}$ & $\begin{array}{l}\text { Média } \\
\text { Quadrática }\end{array}$ & Teste F \\
\hline Regressão & 0,2615 & 9 & 0,0291 & \\
\hline Resíduos & 0,0138 & 8 & 0,0017 & 16,85 \\
\hline $\begin{array}{l}\text { Façta de } \\
\text { ajuste }\end{array}$ & 0,0074 & 5 & 0,0015 & \\
\hline Erro puro & 0,0064 & 3 & 0,0021 & 0,70 \\
\hline Total & 0,2753 & 17 & & \\
\hline
\end{tabular}

$\%$ da variância $=94,99$

\% máxima de variância explicada $=97,69$

Surpreendentemente, verifica-se que os parâmetros quadráticos e de interação não são significativos, ou seja, o melhor modelo para descrever a reação não catalítica nas concentrações estudadas parece ser mesmo o modelo linear. Isso justifica-se já que o modelo obtido, agora a partir de um número maior de experimentos, reduz-se novamente a um modelo linear. Isso pode ser verificado, realizando-se uma nova análise de variância para o ajuste do modelo linear, desta vez incluindo os ensaios adicionais realizados no planejamento estrela, o que é mostrado na Tabela 4.

Tabela 4. Análise da variância para o ajuste do modelo linear para a reação não catalítica, aos ensaios do planejamento estrela.

\begin{tabular}{|l|l|l|l|l|}
\hline $\begin{array}{l}\text { Fonte de } \\
\text { Variação }\end{array}$ & $\begin{array}{l}\text { Soma } \\
\text { Quadrática }\end{array}$ & $\begin{array}{l}\text { Graus de } \\
\text { Liberdade }\end{array}$ & $\begin{array}{l}\text { Média } \\
\text { Quadrática }\end{array}$ & Teste F \\
\hline Regressão & 0,2558 & 3 & 0,0853 & \\
\hline Resíduos & 0,0196 & 14 & 0,0014 & 61,00 \\
\hline $\begin{array}{l}\text { Façta de } \\
\text { ajuste }\end{array}$ & 0,0132 & 11 & 0,0012 & \\
\hline Erro puro & 0,0064 & 3 & 0,0021 & 0,57 \\
\hline Total & 0,2753 & 17 & & \\
\hline \% da variância $=92,89$ & & & \\
\hline
\end{tabular}

$\%$ da variância $=92,89$

\% máxima de variância explicada $=97,69$

Fazendo-se os cálculos necessários, conclui-se que a regressão é significativa na descrição dos resultados e que o modelo não apresenta falta de ajuste. Porém, o mais importante é que a análise visual do gráfico de resíduos, mostrado na Figura 2, não apresenta a tendência verificada anteriormente.

A comparação das Tabelas 2 e 4, para o ajuste do modelo linear para a reação não catalítica, obtidas a partir do planejamento fatorial e do planejamento fatorial estrela, respectivamente, permite verificar que apenas o número de graus de liberdade foi alterado, mas é essencialmente a mesma já que um número maior de ensaios foi empregado nos cálculos. No entanto, a comparação entre as Figuras 1 e 2 permite aceitar o modelo linear sem restrições.

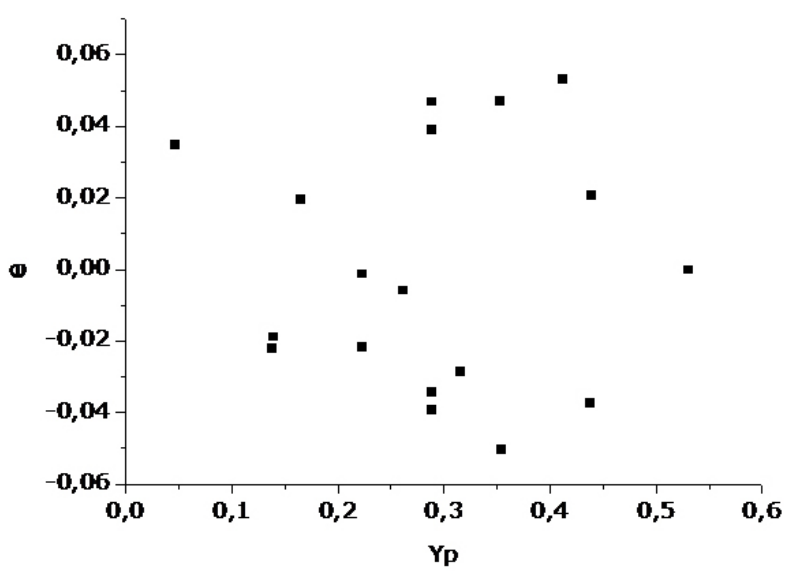

Figura 2 - Gráfico dos resíduos (e) contra as respostas previstas (Yp) para o modelo linear para a reação não catalítica a partir dos dados do planejamento estrela.

A inspeção destas Figuras, juntamente com os resultados apresentados nas Tabelas 2 e 4 , nos permite concluir que o modelo linear é realmente o mais adequado para descrever o efeito dos reagentes $\mathrm{KI}, \mathrm{H}_{2} \mathrm{O}_{2}$ e $\mathrm{H}_{2} \mathrm{SO}_{4}$ na absorbância da reação não catalítica, nas concentrações estudadas. Após o cálculo dos erros dos coeficientes e intervalos de confiança, verifica-se que a equação que descreve a reação não catalítica nestas condições pode ser descrita pela equação 5:

$$
\begin{gathered}
A=0,03( \pm 0,01)+0,11 x_{1}( \pm 0,01)+0,09 x_{2}( \pm 0,01)+ \\
+0,05 x_{3}( \pm 0,01) \quad \text { equação } 5
\end{gathered}
$$

Apesar das equações 4 e 5 diferirem basicamente apenas no coeficiente independente das variáveis testadas, o modelo da equação 5 é válido para uma faixa maior de concentrações, sendo válido entre 0,6 e $3,4 \mathrm{mmol} \mathrm{L}^{-1}$ de $\mathrm{KI} ; 0,4$ e $1,1 \mathrm{mmol} \mathrm{L}^{-1}$ de $\mathrm{H}_{2} \mathrm{O}_{2}$ e 0,012 e $0,068 \mathrm{mmol} \mathrm{L}^{-1}$ de $\mathrm{H}_{2} \mathrm{SO}_{4}$, em relação ao planejamento fatorial inicial que era de 1,0 e $3,0 \mathrm{mmol} \mathrm{L}^{-1}$ de KI; 0,5 e $1,0 \mathrm{mmol} \mathrm{L}^{-1} \mathrm{de}$ $\mathrm{H}_{2} \mathrm{O}_{2}$ e 0,02 e $0,06 \mathrm{mmol} \mathrm{L}^{-1}$ de $\mathrm{H}_{2} \mathrm{SO}_{4}$.

\section{Modelamento da reação catalítica}

Nesse caso, aproveitamos o planejamento estrela para explorar a resposta analítica de interesse $(\Delta \mathrm{A})$ na mesma faixa de concentrações, realizando os experimentos previstos no fatorial estrela também para a reação na presença de molibdênio, ao invés de realizar apenas o planejamento fatorial. Empregando os valores de $\Delta \mathrm{A}$ 
listados na Tabela 1, verificou-se que o modelo linear é adequado para descrever o aumento de absorbância na presença do catalisador, pois num nível de confiança de $95 \%$ a regressão foi signicativa ( $\mathrm{F}$ calculado $(3,14)$ $=76,26$ e $\mathrm{F}$ tabelado $=3,34)$ e não há evidência de falta de ajuste ( $\mathrm{F}$ calculado $(11,3)=2,49$ e $\mathrm{F}$ tabelado =8,7). Porém, verificou-se que o efeito da variável $\mathrm{x}_{3}\left(\mathrm{H}_{2} \mathrm{SO}_{4}\right)$ não é significativo, ou seja o aumento da acidez na faixa estudada não aumenta a produção de iodo na presença de molibdênio, como mostrado na equação 6:

$$
\begin{gathered}
\Delta \mathrm{A}=0,81( \pm 0,02)+0,34 \mathrm{x}_{1}( \pm 0,02)+ \\
+0,08 \mathrm{x}_{2}( \pm 0,01) \quad \text { equação } 6
\end{gathered}
$$

Diferentemente da reação não catalítica, alterações na concentração da variável $\mathrm{x}_{1}(\mathrm{KI})$ são claramente mais importantes para o ganho na resposta analítica do que variações na concentração de peróxido de hidrogênio, isso porque o coeficiente para esta variável é maior e o erro padrão mostrado nas duas equações é comparável. Essas diferenças tornam-se ainda mais evidentes, com o auxílio das superfícies de resposta, se as variáveis graficadas forem selecionadas adequadamente.

Superfície de resposta e condição de ótimo

Superfícies de resposta são gráficos tridimensionais que mostram a variação da resposta experimental em função de alterações nos níveis de duas variáveis selecionadas[4], como por exemplo, os reagentes $\mathrm{KI}$ e $\mathrm{H}_{2} \mathrm{O}_{2}$. Se houver outras variáveis, estas serão fixadas num determinado nível e a equação deverá ser rearranjada para obter-se a respectiva superfície de resposta. No caso das equações 5 e 6, a variável $x_{3}$ foi fixada no nível zero (em termos escalonados), correspondente ao ponto central, mas poderia ter sido fixada em qualquer outro nível de interesse. O uso do nível zero não significa, no entanto, que o ácido sulfúrico não foi adicionado, muito pelo contrário, no nível zero a concentração adicionada foi de $0,04 \mathrm{~mol} \mathrm{L^{- }}$ ${ }^{1}$, mas o que acontece é que a equação fica simplificada e permite uma representação em três dimensões. Nas figuras apresentadas são mostradas ainda as chamadas "curvas de nível", que são na verdade uma projeção bidimensional das mesmas superfícies de resposta.

A escala da Figura 3 (A e B) deixa clara a diferença de absorbância entre as reações de produção de iodo na presença e na ausência de molibdênio. Como, a partir dos dados da Tabela 1, podemos verificar que o aumento em alguma das variáveis não irá aumentar o valor da resposta analítica, não será necessário realizar uma ascensão ao máximo para localizar as condições experimentais ótimas, pois essas já estão incluídas no nosso planejamento. Basta agora selecionar a condição em que a diferença entre as respostas dos modelos ajustados é máxima, isso pode ser feito empregando as equações 5 e 6 diretamente (essa comparação é facilitada pois o erro padrão dos coeficientes do modelo é muito próximo), ou com o auxílio da Figura 3.

A condição experimental mais favorável para a reposta analítica é empregar a menor concentração da variável $x_{3}$ (ácido sulfúrico) já que no intervalo testado, o aumento nos níveis dessa variável só aumenta a produção de iodo da reação não catalítica. Assim, podemos empregar o nível -1 (que corresponde a $0,02 \mathrm{~mol} \mathrm{~L}^{-1}$ ). Outra possibilidade é elevar os níveis da variável que mais contribui para o aumento da resposta analítica (maior coeficiente na equação ou maior inclinação a superfície de resposta). Nesse caso, trata-se da variável $\mathrm{x}_{1}(\mathrm{KI})$, que pode ser fixada em +1 (que corresponde a 3,0 $\mathrm{mmol} \mathrm{L}^{-1}$ ). Essa condição não é a ideal, pois a absorbância da reação não catalítica também aumenta, ainda que com menor intensidade, mas não nos resta outra alternativa. Já a variável $x_{2}$ $\left(\mathrm{H}_{2} \mathrm{O}_{2}\right)$ apresenta praticamente a mesma contribuição nos dois modelos, sendo impossível definir uma condição claramente favorável à resposta analítica. Dessa forma, poderíamos fixá-la no nível -1, pretendendo minimizar a absorbância da reação catalítica da melhor maneira possível, mas optamos por fixá-la no nível zero (ponto central, correspondente a $0,75 \mathrm{mmol} \mathrm{L}^{-1}$ ) para manter $\Delta \mathrm{A}$ o mais próximo de 1.000 unidade de absorbância.

O processo de otimização está concluído, mas usualmente costuma-se verificar as figuras de mérito da condição otimizada e para poder compará-la com outras condições experimentais utilizadas na literatura[10]. Para a determinação catalítica de $\mathrm{Mo}(\mathrm{VI})$, podemos preparar uma curva de calibração, variando apenas a concentração de $\mathrm{Mo}(\mathrm{VI})$ adicionada ao meio reacional. Esta deve ser preparada numa faixa ampla de concentração para permitir a determinação de sua faixa linear, o limite de deteç̧ão e outros parâmetros típicos da calibração como o coeficiente linear e a equação da regressão linear típica. Assim, verificou-se que a curva de calibração obtida foi linear entre 10 e $60 \mu \mathrm{g} \mathrm{L}^{-1}$ de $\mathrm{Mo}(\mathrm{VI})$. Uma equação da curva de calibração típica foi: $\Delta \mathrm{A}=0,61+0,026[\mathrm{Mo}(\mathrm{VI})]$, com um coeficiente de correlação $r=0,9976$, cujo limite de detecção foi estimado em de $8 \mu g \mathrm{~L}^{-1}$. 

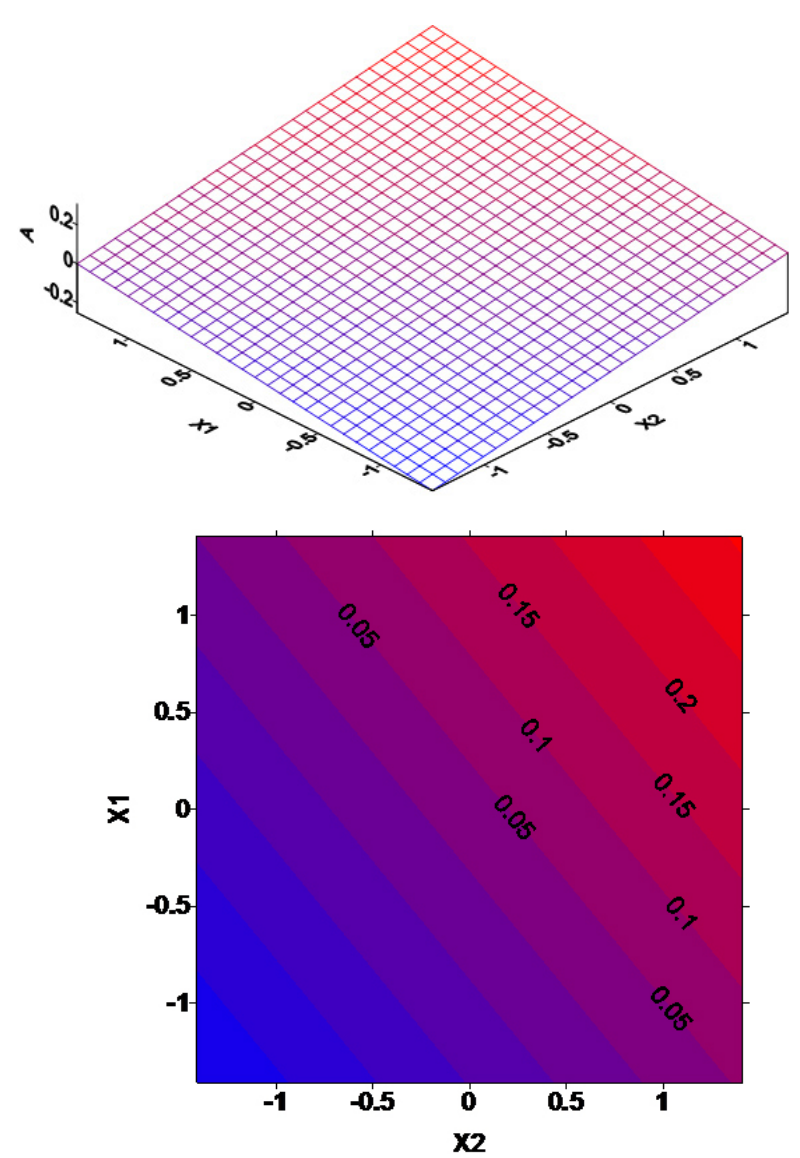

Figura 3A - Superfície de resposta e curvas de nível para reação não catalítica
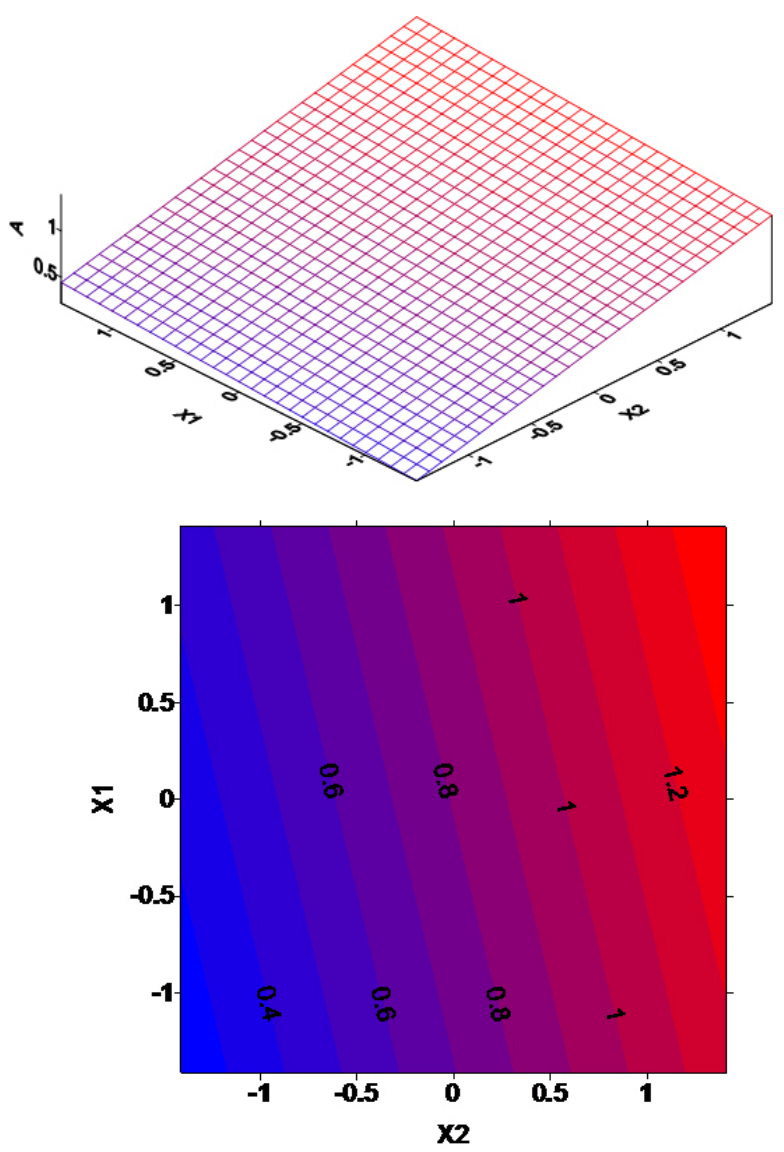

Figura 3B - Superfície de resposta e curvas de nível para o delta.
Referências Bibliográficas

1. "Métodos de Otimização em Quimica", In: Planejamento Experimental e Otimização, em http:// www.chemkeys.com. Acessado em 15/09/2005.

2. Bruns,R.E.; Andrade,J.C. de ;Reis,C. e Nakai,D., "Is statistical lack of fit a reliable criterion for chemical complexity?”, Chemom. Intell. Lab Sys., 1996, 33: 159-166.

3. Barros Neto, B.; Scarmínio, I.S.; Bruns, R.E., Como Fazer Experimentos: Pesquisa e Desenvolvimento na Ciência e na Indústria. Editora da Unicamp: Campinas, 2002.

4. Box, G. E. P.; Hunter, W. G.; Hunter, I. S., In: Statistics for Experimenters: an Introduction to Design, Data Analysis and Model Building; Bradley, R. A., Hunter, J. S., Kendall, D. G., and Watson, G. S., (Eds.); Wiley Series in Probability and Mathematical Statistics, Wiley: New York, Cap. 10, 1978

5. Yatsimirskii, K. B., In Kinetic Methods of Analysis; Belcher, R.; Gordon, L. (Eds.); International Series of Monographs in Analytical Chemistry, Pergamon Press: London, 1966.

6. Piper, C. S.; Beckwith, R. S., "A new method for determination of small amounts of molybdenum in plants”, J. Soc. Chem. Ind., 1948, 67: 374-379.

7. Hadjiioannou, T. P., "Microdetermination of molybdenum by an automatic reaction-rate method", Anal. Chim. Acta., 1966, 35(3): 360-364.

8. de Andrade, J. C.; Eiras, S. P; Bruns, R. E., "Study of the molybdenum(VI) catalytic response in the oxidation of iodide by hydrogen peroxide using a monosegmented continuous-flow system", Anal. Chim. Acta., 1991, 255(1): 149-155.

9. Baccan, N.; de Andrade, J.C.; Godinho, O. E. S.; Barone, J. S., Química analítica quantitativa elementar. $3^{a}$ ed. revisada, 3a reimpressão, São Paulo: Edgar Blücher, 2005.

10. Analytical Methods Committee, "Recommendations for the definition, estimation and use of the detection limit", Analyst., 1987, 112: 199-204. 